OPEN ACCESS

Edited by:

David Pasquier,

Oscar Lambret Centre, France

Reviewed by: Igor Latorzeff,

Clinique Pasteur, France Vanessa Figlia,

IRCCS Sacro Cuore Don Calabria, Italy

*Correspondence:

Albert J. Chang ajchang@mednet.ucla.edu

Specialty section: This article was submitted to

Radiation Oncology,

a section of the journal

Frontiers in Oncology

Received: 12 January 2022 Accepted: 07 February 2022 Published: 08 March 2022

Citation: Kundu P, Lin EY, Yoon SM, Parikh NR, Ruan D, Kishan AU, Lee A, Steinberg ML and Chang AJ (2022) Rectal Radiation Dose and Clinical Outcomes in Prostate Cancer Patients

Treated With Stereotactic Body Radiation Therapy With and Without Hydrogel.

Front. Oncol. 12:853246. doi: 10.3389/fonc.2022.853246

\section{Rectal Radiation Dose and Clinical Outcomes in Prostate Cancer Patients Treated With Stereotactic Body Radiation Therapy With and Without Hydrogel}

\author{
Palak Kundu ${ }^{1}$, Eric Y. Lin ${ }^{1,2}$, Stephanie M. Yoon ${ }^{1}$, Neil R. Parikh ${ }^{1}$, Dan Ruan ${ }^{1}$, \\ Amar U. Kishan ${ }^{1}$, Alan Lee ${ }^{1}$, Michael L. Steinberg ${ }^{1}$ and Albert J. Chang ${ }^{1 *}$ \\ 1 Department of Radiation Oncology, David Geffen School of Medicine at UCLA, Los Angeles, CA, United States, \\ ${ }^{2}$ David Geffen School of Medicine at UCLA, Los Angeles, CA, United States
}

Background: Patients with prostate cancer treated with stereotactic body radiation therapy (SBRT) may experience gastrointestinal (GI) toxicity. The hydrogel may mitigate this toxicity by reducing the rectal radiation dose. The purpose of this study is to compare rectal radiation dose and Gl toxicity in patients receiving prostate SBRT with and without hydrogel.

Methods: Consecutive patients treated with SBRT between February 2017 and January 2020 with and without hydrogel were retrospectively identified. Baseline characteristics including prostate volume, rectal diameter, body mass index (BMI), age, pretreatment prostate-specific antigen (PSA), Gleason score, T-stage, and androgen deprivation therapy (ADT) usage were compared. Dosimetric outcomes (V40Gy, V36Gy, V32Gy, V38Gy, and V20Gy), rates of acute ( $\leq 90$ days) and late (>90 days) Gl toxicity, and PSA outcomes were evaluated for patients with and without hydrogel.

Results: A total of 92 patients were identified (51 hydrogel and 41 non-hydrogel). There were no significant differences in baseline characteristics. Rectal V38(cc) was significantly less in the hydrogel group (mean $0.44 \mathrm{vs}$. mean $1.41 \mathrm{cc}, \mathrm{p}=0.0002$ ), and the proportion of patients with $\mathrm{V} 38(\mathrm{cc})<2 \mathrm{cc}$ was greater in the hydrogel group ( $92 \%$ vs. $72 \%, p=0.01$ ). Rectal dose was significantly lower for all institutional dose constraints in the hydrogel group ( $p<0.001$ ). The hydrogel group experienced significantly less acute overall $\mathrm{Gl}$ toxicity (16\% hydrogel vs. $28 \%$ non-hydrogel, $p=0.006$ ), while the difference in late $\mathrm{Gl}$ toxicity trended lower with hydrogel but was not statistically significant (4\% hydrogel vs. $10 \%$ non-hydrogel, $p=0.219$ ). At a median follow-up of 14.8 months, there were no biochemical recurrences in either group.

Conclusion: Hydrogel reduces rectal radiation dose in patients receiving prostate SBRT and is associated with a decreased rate of acute Gl toxicity.

Keywords: hydrogel, prostate cancer, SBRT (stereotactic body radiation therapy), radiation oncology, outcomes 


\section{INTRODUCTION}

Stereotactic body radiation therapy (SBRT) is a recommended treatment for prostate cancer and is increasingly utilized $(1,2)$. This technique, which utilizes ultra-hypofractionated radiation regimens ( $\geq 5$ Gy per fraction), is now standard of care and has been suggested to be non-inferior to standard fractionation radiation for biochemical and local control (3). Additionally, ultra-hypofractionated treatment courses with SBRT, which require only 5-7 visits, are significantly more convenient for patients. However, gastrointestinal (GI) toxicity remains an issue for prostate SBRT. For example, the PACE-B trial reported 53\% Grade 1, 10\% Grade 2, and < 1\% Grade 3 Radiation Therapy Oncology Group (RTOG) GI toxicities (4). The rectum is adjacent to and often abuts the prostate and thus may receive significant incidental radiation leading to GI toxicity. Acute radiation-related rectal toxicity can occur due to inflammation, fibrosis, microvascular damage, and edema within the bowel wall and mucosa $(5,6)$. Late sequelae may include bleeding, urgency, and incontinence, which can be predicted by radiation volumetric dose parameters (7-10).

To limit radiation dose to the rectum, various methods have been employed to create space between the prostate and rectum, including collagen or hyaluronic acid injection, and biodegradable rectal spacer balloons (11-13). Another such method is the injection of the hydrogel into Denonvilliers' fascia between the rectum and prostate. This hydrogel is biologically inert and composed of two liquids that mix postinjection to polymerize and solidify within the patient (Figure 1). Hydrogel has been shown to reduce rectal dose in patients receiving standard fractionation radiation therapy (14).

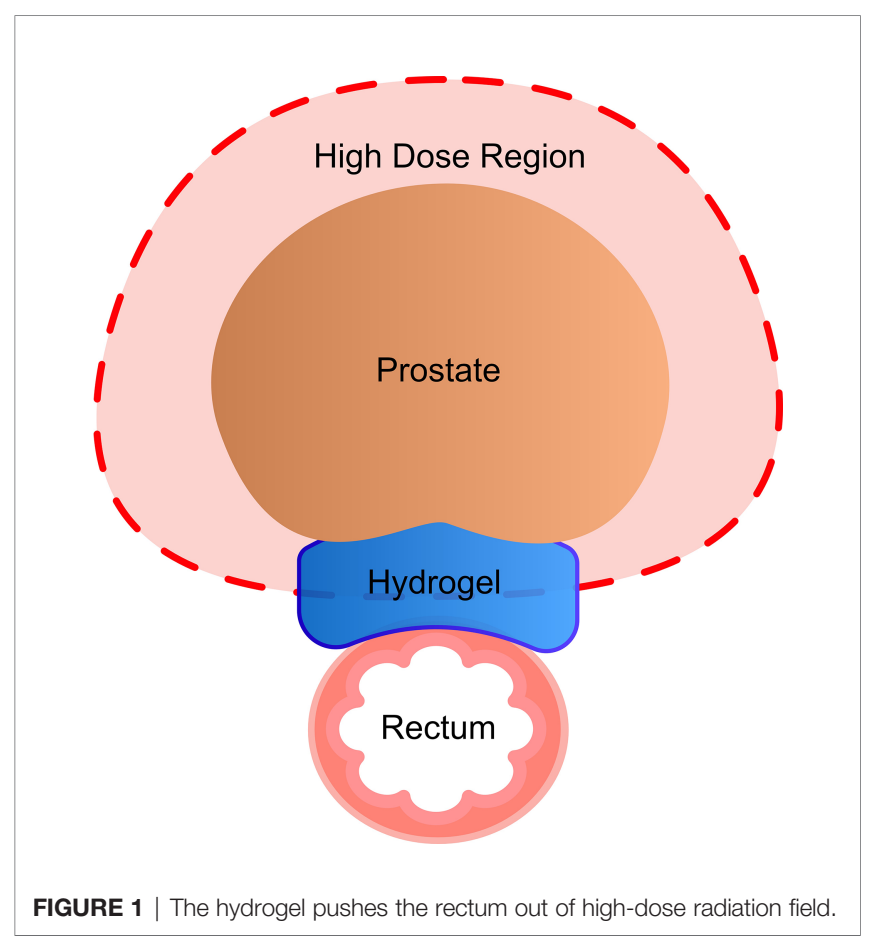

However, data on the safety, efficacy, and clinical outcomes of hydrogel in patients receiving SBRT are limited.

The purpose of this study is to compare rectal dose and associated GI toxicity with or without hydrogel in patients with prostate cancer undergoing SBRT to the prostate (Boston Scientific, Marlborough, MA, USA).

\section{METHODS}

This Institutional Review Board-approved retrospective study included patients who received SBRT for treatment of localized prostate cancer at a single academic institution between February 2017 and January 2020. All patients were aged 18 years or older and did not receive prior pelvic radiation, transurethral resection of the prostate, or any other focal treatment.

Hydrogel was offered to all patients without posterior extracapsular extension (ECE) on MRI. For patients receiving hydrogel, Denonvilliers' space was approached transperineally with a 17-gauge needle and was gently hydrodissected with 10 $\mathrm{cm}^{3}$ of $0.9 \%$ normal saline under transrectal ultrasound guidance. Upon confirmation of Denonvilliers' space expansion and separation of Denonvilliers' fascia from the rectal wall, $10 \mathrm{~cm}^{3}$ of hydrogel was administered into this space. All patients underwent pretreatment multiparametric MRI at diagnosis and CT simulation for SBRT treatment planning. Patients who received hydrogel also subsequently underwent MRI within 1 week of CT simulation. All patients received linear accelerator-based radiation treatment with $40 \mathrm{~Gy}$ in 5 fractions to the clinical target volume (CTV), which was defined as the prostate and proximal seminal vesicles. The CTV was expanded by $5 \mathrm{~mm}$ in all directions, except $3-4 \mathrm{~mm}$ posteriorly to form the planning target volume (PTV). The treatment dose was prescribed such that $95 \%$ of the PTV received the prescription dose, and the institutional dose constraints were rectum V20Gy $\leq 50 \%$, V32Gy $\leq 20 \%$, $\mathrm{V} 36 \mathrm{~Gy} \leq 10 \%$, and $\mathrm{V} 40 \mathrm{~Gy} \leq 5 \%$; bladder $\mathrm{V} 20 \mathrm{~Gy} \leq 40 \%$ and $\mathrm{V} 40 \mathrm{~Gy} \leq 10 \%$; and small bowel V20Gy $<30 \mathrm{cc}$ and D0.035cc $\leq$ 35 Gy.

Baseline characteristics including age, body mass index (BMI), prostate volume, rectal diameter, T-stage, Gleason Grade Group, pretreatment prostate-specific antigen (PSA), and androgen deprivation therapy (ADT) use were collected. Risk categories were defined according to the National Comprehensive Cancer Network (NCCN). Prostate volume was assessed on pretreatment MRI, and rectal diameter was measured as the largest diameter at the mid-gland level of the prostate on the CT simulation scan.

The age, BMI, prostate volume, rectal diameter, and pretreatment PSA between the hydrogel and non-hydrogel patients were compared by Student's or Welch's t-test. The Gleason scores and risk groups were compared using the Kruskal-Wallis test, while T-stage and ADT use were compared using Fisher's exact test.

Rectal dose-volume histogram (DVH) parameters corresponding to institutional dose constraints (rectum V40Gy, 
V36Gy, V32Gy, and V20Gy) and V38Gy(cc), which has previously been shown to predict high-grade late hematochezia, were collected (15). Differences between the hydrogel and non-hydrogel patients in rectal dose parameters were compared using t-test for two-sample mean when variances between groups were equal and Welch's test when unequal, and the proportion of patients with $\mathrm{V} 38 \mathrm{~Gy}<2 \mathrm{cc}$ was compared using Fisher's exact test. The highest reported Common Terminology Criteria for Adverse Events (CTCAE) for acute ( $\leq 90$ days) and late (> 90 days) GI toxicity scores reported during follow-up were collected and compared using Fisher's exact test. Posttreatment PSAs were collected to evaluate the incidence of biochemical recurrence per Phoenix definition (PSA nadir $+2 \mathrm{ng} / \mathrm{ml})$.

\section{RESULTS}

A total of 92 localized prostate cancer patients were identified who underwent SBRT, of whom 51 patients received hydrogel. Baseline characteristics are shown in Table 1, and no significant differences were observed (Table 1). The median overall followup was 14.8 months (range 3.8-41.5 months; hydrogel median
14.8 months, non-hydrogel median 16.2 months), and the median age was 72 years (range 46-85). Included in the study were 20 high-risk, 65 intermediate-risk, and 7 low-risk patients defined by NCCN criteria. A trend towards NCCN high-risk group disease in non-hydrogel patients and towards unfavorable intermediate-risk group disease in hydrogel patients was observed but was not statistically significant. A total of 3 patients ( 2 hydrogel patients) had T3a disease. None of these patients had posterior ECE on imaging. A total of four patients (1 hydrogel patient) had T3b disease. Androgen deprivation therapy was given to $39 \%$ and $35 \%$ of the hydrogel and nonhydrogel patients, respectively. The median time from hydrogel placement to SBRT was 10 days (range 4-25 days). At a median follow-up of 14.8 months in the hydrogel group, there were no biochemical recurrences.

Rectal dose was significantly lower for all evaluated radiation dose parameters in the hydrogel group (Figure 2). The greatest relative differences were seen in the high dose parameters; i.e., V40Gy was 7 -fold less in the hydrogel group $(0.18 \%$ vs. $1.30 \%)$. Additionally, rectal V38(cc) was significantly less in the hydrogel group (mean 0.44 vs. mean $1.41 \mathrm{cc}, \mathrm{p}=0.0002$ ), and the proportion of patients with $\mathrm{V} 38(\mathrm{cc})<2 \mathrm{cc}$ was greater in the hydrogel group ( $92 \%$ vs. $72 \%, \mathrm{p}=0.01)$.

TABLE 1 | Baseline patient clinical characteristics.

\begin{tabular}{|c|c|c|c|}
\hline \multirow[b]{2}{*}{ Characteristics } & No hydrogel $(n=41)$ & \multirow[t]{2}{*}{ Hydrogel $(n=51)$} & \multirow[t]{2}{*}{ p-Value } \\
\hline & Number of patients (\%) & & \\
\hline Age & & & 0.77 \\
\hline$\leq 60$ & $6(15 \%)$ & $6(12 \%)$ & \\
\hline $61-70$ & $12(29 \%)$ & $15(29 \%)$ & \\
\hline \multirow[t]{2}{*}{$\geq 70$} & $23(56 \%)$ & $30(59 \%)$ & \\
\hline & Median = 71 (range 46-85) & Median $=72($ range $52-85)$ & \\
\hline BMI $\left(k g / m^{2}\right)$ & Mean $=26.9$, median $=26.4($ range $20.2-45.0)$ & Mean $=26.7$, median $=26.4($ range $16.1-35.6)$ & 0.77 \\
\hline Stage & & & 0.70 \\
\hline $\mathrm{T} 1-\mathrm{T} 2$ & $37(90 \%)$ & $48(94 \%)$ & \\
\hline T3 and above & $4(10 \%)$ & $3(6 \%)$ & \\
\hline Grade group & & & 0.81 \\
\hline 1 & $3(7 \%)$ & $5(10 \%)$ & \\
\hline 2 & $17(41 \%)$ & $16(32 \%)$ & \\
\hline 3 & $11(27 \%)$ & $22(44 \%)$ & \\
\hline 4 & $5(12 \%)$ & 5 (10\%) & \\
\hline 5 & $5(12 \%)$ & $2(4 \%)$ & \\
\hline Pretreatment PSA (ng/ml) & & & 0.82 \\
\hline$<10$ & $27(66 \%)$ & $40(78 \%)$ & \\
\hline $10-20$ & $11(27 \%)$ & $10(20 \%)$ & \\
\hline \multirow[t]{2}{*}{$>20$} & $3(7 \%)$ & $1(2 \%)$ & \\
\hline & Mean $=11.4$, median $=7.64,($ range $2.5-77)$ & Mean = 12.6, median = $7.1($ range 0.9-254.4 $)$ & \\
\hline ADT & & & 0.83 \\
\hline Yes & 16 (39\%) & $18(35 \%)$ & \\
\hline \multirow[t]{2}{*}{ No } & $25(61 \%)$ & $31(65 \%)$ & \\
\hline & Median = 6 months, (range 3-24 months) & Median = 6 months, (range 1.5-24 months) & \\
\hline NCCN risk category & & & 0.25 \\
\hline Low & $2(5 \%)$ & 5 (10\%) & \\
\hline Favorable intermediate & $13(32 \%)$ & $10(20 \%)$ & \\
\hline Unfavorable intermediate & $14(34 \%)$ & $28(55 \%)$ & \\
\hline High & $12(29 \%)$ & $8(16 \%)$ & \\
\hline Prostate volume (cc) & Mean $=56.6$, median $=52.2($ range $27.3-112.3)$ & Mean $=49.1$, median $=45.7($ range $16.5-86.8)$ & 0.07 \\
\hline Rectal diameter (cm) & Mean $=3.7$, median $=3.5($ range $2.4-5.3)$ & Mean $=3.6$, median $=3.5($ range $2.3-5.6)$ & 0.54 \\
\hline
\end{tabular}

BMI, body mass index; PSA, prostate-specific antigen; ADT, androgen deprivation therapy; NCCN, National Comprehensive Cancer Network. 


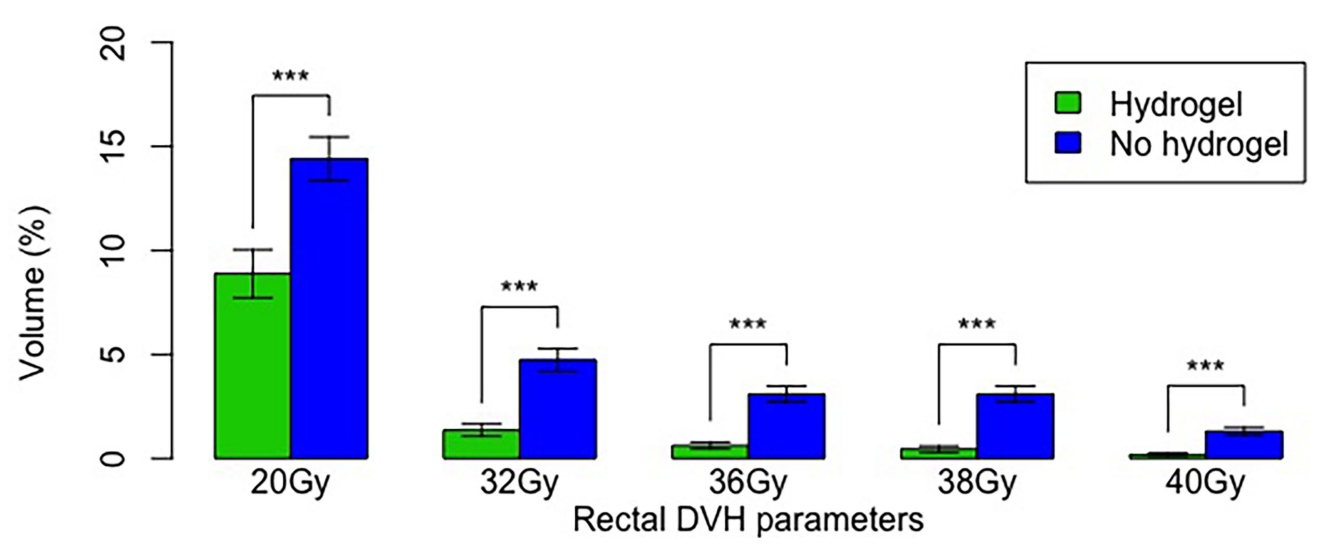

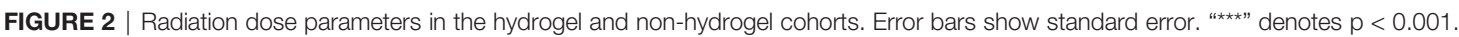

TABLE 2 | Acute Gl toxicity rates.

\begin{tabular}{lcc}
\hline & Non-hydrogel \% (n) & Hydrogel \% (n) \\
\hline Grade 1 & $24 \%(10)$ & $12 \%(6)$ \\
Grade 2 & $2 \%(1)$ & $4 \%(2)$ \\
Grade 3 & $2 \%(1)$ & $0 \%(0)$ \\
\hline
\end{tabular}

Gl, gastrointestinal.

The rates of acute Grade 1, 2, and 3 GI toxicities are shown in Table 2. Overall, the non-hydrogel group had greater acute GI toxicity ( $\mathrm{p}=0.006)$, including rectal urgency (4), constipation (2), and diarrhea (4). While most of the toxicities were Grade 1, one case of constipation was Grade 2, and one case of diarrhea was Grade 3, which later resolved. Of note, 6 minor acute Grade 1 adverse events resulting from the procedure were reported in the current cohort, and all resolved, including constipation (2), loose stools (1), and minimal or unspecified GI symptoms (3). The highest reported late GI toxicity was Grade 1 (diarrhea in all cases): 2 patients (4\%) in the hydrogel group and 4 patients $(10 \%)$ in the non-hydrogel group. This difference in late GI toxicity was not statistically significant $(p=0.219)$.

\section{DISCUSSION}

Hydrogel significantly reduced the relevant radiation volumetric dose parameters by creating a physical separation between the prostate and rectum and thereby displacing the rectum from the high dose radiation field. The procedure was safe and well tolerated with no short- or long-term procedural-related sequelae. Furthermore, hydrogel was associated with a significant reduction in acute GI toxicity. We did not observe a similar association for late GI toxicity; however, more events may occur with longer follow-up. The acute diarrhea reported in the hydrogel group may also be due to hydrogel, and not radiation, given that hydrogel may irritate the rectum and that patients are often prescribed a stool softener to prevent constipation.
Importantly, there were no differences in biochemical recurrence, indicating oncologic outcomes were not compromised. However, the median overall follow-up time was only 14.8 months. Furthermore, there were only three patients with ECE (none were posterior) and four patients with seminal vesicle invasion. Therefore, caution should be exercised for T3-T4 patients with posterior ECE or invasion of the rectum out of theoretical concern that gross disease may be displaced out of the intended treatment field. Therefore, pretreatment MRI is recommended to assess disease extent posteriorly.

Though data on the effects of hydrogel in patients receiving SBRT are limited, the dose reduction observed in this study is consistent with a previous study of hydrogel with dose-escalated standard fractionation radiation (14), which similarly showed the greatest relative reductions in the high dose volumetric parameters; i.e., V82Gy was also 7-fold less in the hydrogel group $(0.2 \%$ vs. $1.3 \%)$. Though no difference in acute GI toxicity was reported, late Grade 1 toxicity was less frequent in the hydrogel group (16.6\% vs. $41.8 \%)$. Another study of hydrogel with ultra-hypofractionation without a comparative nonhydrogel group showed similar rates of acute GI toxicity (16\% Grade 1 and $4 \%$ Grade 2) and no difference in late rectal toxicity (16). Studies of MRI-guided, daily adaptive SBRT similarly show reduced rectal dose and reduced intra-fraction motion and importantly collected patient-reported outcomes that did not show decreased quality of life in patients receiving hydrogel spacers $(17,18)$. The acute benefit of hydrogel may be more pronounced for ultra-hypofractionation than for standard fractionation, especially given the concern for the worse acute quality of life for ultra-hypofractionation seen in the HYPO-RTPC trial (19). Acute Bowel Quality of Life was worse at $<3$ months but the same at 3 months. Furthermore, the HYPO-RTPC SBRT arm reported 9.4\% acute Grade $2+$ and $2.2 \%$ late 2 -year Grade 2+ GI toxicity rates. The higher rate of Grade 2+ toxicity on HYPO-RT-PC compared to the current study may be due to the use of older radiation techniques. Additionally, the main rectal dose constraint used in the HYPO-RT-PC trial was V90\% $\leq 15 \%$, while our corresponding institutional constraint was 
V36Gy $\leq 10 \%$. A phase II trial of SBRT at our institution demonstrated that acute and late Grade 2 GI toxicities were $3.3 \%$ and $3.9 \%$, respectively (20).

It is imperative that the risks and benefits of hydrogel be considered prior to its administration. Hall et al. queried the Food and Drug Administration (FDA) Manufacturer and User Facility Design Database (MAUDE) and noted 85 adverse events related to hydrogel placement, of which $69 \%$ were scored as grade $\geq 3$ toxicity including descriptions of colostomy, anaphylactic events, rectal injection pulmonary emboli, and death (21). There is no doubt that these events are severe, but in relation to the total number of hydrogel cases performed $(109,165$ estimated), these events are rare $(0.07 \%)$. Of note, adequate training and experience are critical to ensure the safety of hydrogel administration, and physicians must be credentialed to perform this procedure.

The current study has several limitations, one of which is that this is a retrospective analysis of non-randomized patients with a contemporary control used instead. Furthermore, physician preference and insurance reimbursement may have driven the decision patient decision to pursue hydrogel, and these confounders may be correlated with toxicity outcomes. Hydrogel patients were only simulated once without a separate plan to compare DVH parameters without hydrogel within the same patient. Furthermore, physician-reported toxicity may underestimate the true incidence of GI toxicities, and late GI

\section{REFERENCES}

1. Malouff TD, Stross WC, Seneviratne DS, Waddle MR, May BC, Buskirk SJ, et al. Current Use of Stereotactic Body Radiation Therapy for Low and Intermediate Risk Prostate Cancer: A National Cancer Database Analysis. Prostate Cancer Prostatic Dis (2020) 23:349-55. doi: 10.1038/s41391-0190191-9

2. NCCN Guidelines (2021). Available at: https://www.nccn.org/professionals/ physician_gls/pdf/prostate.pdf (Accessed 30 June 2021).

3. Widmark A, Gunnlaugsson A, Beckman L, Thellenberg-Karlsson C, Hoyer M, Lagerlund $M$, et al. Ultra-Hypofractionated Versus Conventionally Fractionated Radiotherapy for Prostate Cancer: 5-Year Outcomes of the HYPO-RT-PC Randomised, non-Inferiority, Phase 3 Trial. Lancet (2019) 394(10196):385-95. doi: 10.1016/S0140-6736(19)31131-6

4. Brand DH, Tree AC, Ostler P, van der Voet H, Loblaw A, Chu W, et al. Intensity-Modulated Fractionated Radiotherapy Versus Stereotactic Body Radiotherapy for Prostate Cancer (PACE-B): Acute Toxicity Findings From an International, Randomised, Open-Label, Phase 3, non-Inferiority Trial. Lancet Oncol (2019) 20(11):1531-43. doi: 10.1016/S1470-2045(19)30569-8

5. Michalski JM, Gay H, Jackson A, Tucker SL, Deasy JO. Radiation DoseVolume Effects in Radiation-Induced Rectal Injury. Int J Radiat Oncol Biol Phys (2010) 76:S123-9. doi: 10.1016/j.ijrobp.2009.03.078

6. Shadad AK, Sullivan FJ, Martin JD, Egan LJ. Gastrointestinal Radiation Injury: Symptoms, Risk Factors and Mechanisms. World J Gastroenterol (2013) 19(2):185-98. doi: 10.3748/wjg.v19.i2.185

7. Krol R, Smeenk RJ, van Lin ENJT, Hopman WPM. Impact of Late Anorectal Dysfunction on Quality of Life After Pelvic Radiotherapy. Int J Colorectal Dis (2012) 28(4):519-26. doi: 10.1007/s00384-012-1593-5

8. Pollack A, Zagars GK, Starkschall G, Antolak JA, Lee JJ, Huang E, et al. Prostate Cancer Radiation Dose Response: Results of the M. D. Anderson Phase III Randomized Trial. Int J Radiat Oncol Biol Phys (2002) 53(5):1097105. doi: 10.1016/S0360-3016(02)02829-8

9. Peeters STH, Lebesque JV, Heemsbergen WD, van Putten WLJ, Slot A, Dielwart MFH, et al. Localized Volume Effects for Late Rectal and Anal
Grade 2+ GI toxicity occurs with a mean time of 1.5 years posttreatment, which exceeds the median follow-up of the current study $(22,23)$.

\section{CONCLUSION}

In prostate cancer patients treated with SBRT, hydrogel is well tolerated, reduced key rectal dose parameters, and is associated with lower rates of acute GI toxicity.

\section{DATA AVAILABILITY STATEMENT}

The data analyzed in this study is subject to the following licenses/restrictions: due to the nature of this research and protected health information, participants of this study did not agree for their data to be shared publicly. Requests to access these datasets should be directed to AC, ajchang@mednet.ucla.edu.

\section{AUTHOR CONTRIBUTIONS}

All authors contributed equally to the writing and preparation of this manuscript.

Toxicity After Radiotherapy for Prostate Cancer. Int J Radiat Oncol Biol Phys (2006) 64(4):1151-61. doi: 10.1016/j.ijrobp.2005.10.002

10. Vargas C, Martinez A, Kestin LL, Yan D, Grills I, Brabbins DS, et al. DoseVolume Analysis of Predictors for Chronic Rectal Toxicity After Treatment of Prostate Cancer With Adaptive Image-Guided Radiotherapy. Int J Radiat Oncol Biol Phys (2005) 62(5):1297-308. doi: 10.1016/j.ijrobp.2004.12.052

11. Gez E, Cytron S, Yosef RB, London D, Corn BW, Alani S, et al. Application of an Interstitial and Biodegradable Balloon System for Prostate-Rectum Separation During Prostate Cancer Radiotherapy: A Prospective MultiCenter Study. Radiat Oncol (2013) 8(1):96. doi: 10.1186/1748-717X-8-96

12. Noyes WR, Hosford CC, Schultz SE. Human Collagen Injections to Reduce Rectal Dose During Radiotherapy. Int J Radiat Oncol Biol Phys (2012) 82 (5):1918-22. doi: 10.1016/j.ijrobp.2011.02.034

13. Wilder RB, Barme GA, Gilbert RF, Holevas RE, Kobashi LI, Reed RR, et al. Cross-Linked Hyaluronan Gel Improves the Quality of Life of Prostate Cancer Patients Undergoing Radiotherapy. Brachytherapy (2011) 10(1):44-50. doi: 10.1016/j.brachy.2009.12.005

14. Whalley D, Hruby G, Alfieri F, Kneebone A, Eade T. SpaceOAR Hydrogel in Dose-Escalated Prostate Cancer Radiotherapy: Rectal Dosimetry and Late Toxicity. Clin Oncol (2016) 28(10):e148-54. doi: 10.1016/j.clon.2016.05.005

15. Musunuru HB, Davidson M, Cheung P, Vesprini D, Liu S, Chung H, et al. Predictive Parameters of Symptomatic Hematochezia Following 5-Fraction Gantry-Based SABR in Prostate Cancer. Int J Radiat Oncol Biol Phys (2016) 94 (5):1043-51. doi: 10.1016/j.ijrobp.2015.12.010

16. Hwang ME, Mayeda M, Liz M, Goode-Marshall B, Gonzalez L, Elliston CD, et al. Stereotactic Body Radiotherapy With Periprostatic Hydrogel Spacer for Localized Prostate Cancer: Toxicity Profile and Early Oncologic Outcomes. Radiat Oncol (2019) 14(136):1-9. doi: 10.1186/s13014-019-1346-5

17. Alongi F, Rigo M, Figlia V, Cuccia F, Giaj-Levra N, Nicosia L, et al. Rectal Spacer Hydrogel in 1.5T MR-Guided and Daily Adapted SBRT for Prostate Cancer: Dosimetric Analysis and Preliminary Patient-Reported Outcomes. $\mathrm{Br}$ J Radiol (2021) 94(1117):20200848. doi: 10.1259/bjr.20200848

18. Mazzola R, Sicignano G, Cuccia F, Vitale C, Rigo M, Giaj-Levra N, et al. Impact of Hydrogel Peri-Rectal Spacer Insertion on Prostate Gland Intra- 
Fraction Motion During 1.5 T MR-Guided Stereotactic Body Radiotherapy. Radiat Oncol (2020) 15(1):178. doi: 10.1186/s13014-020-01642-z

19. Fransson P, Nilsson P, Gunnlaugsson A, Beckman L, Tavelin B, Norman D, et al. Ultra-Hypofractionated Versus Conventionally Fractionated Radiotherapy for Prostate Cancer (HYPO-RT-PC): Patient-Reported Quality-of-Life Outcomes of a Randomised, Controlled, Non-Inferiority, Phase 3 Trial. Lancet Oncol (2021) 22(2):235-45. doi: 10.1016/S1470-2045 (20)30581-7

20. Kishan AU, Dang A, Katz AJ, Mantz CA, Collins SP, Aghdam N, et al. LongTerm Outcomes of Stereotactic Body Radiotherapy for Low-Risk and Intermediate-Risk Prostate Cancer. JAMA Netw Open (2019) 2(2):1-3. doi: 10.1001/jamanetworkopen.2018.8006

21. Hall WA, Tree AC, Dearnaley D, Parker CC, Prasad V, Roach M 3rd, et al. Considering Benefit and Risk Before Routinely Recommending SpaceOAR. Lancet Oncol (2021) 22(1):11-3. doi: 10.1016/S1470-2045(20)30639-2

22. Zelefsky MJ, Levin EJ, Hunt M, Yamada Y, Shippy AM, Jackson A, et al. Incidence of Late Rectal and Urinary Toxicities After Three-Dimensional Conformal Radiotherapy and Intensity-Modulated Radiotherapy for Localized Prostate Cancer. Int J Radiat Oncol Biol Phys (2008) 70(4):11249. doi: 10.1016/j.ijrobp.2007.11.044

23. Odrazka K, Dolezel M, Vanasek J, Vaculikova M, Zouhar M, Sefrova J, et al. Time Course of Late Rectal Toxicity After Radiation Therapy for Prostate
Cancer. Prostate Cancer Prostatic Dis (2010) 13(2):138-43. doi: 10.1038/ pcan.2009.56

Conflict of Interest: AC received consulting fees and lecture payments from Boston Scientific.

The remaining authors declare that the research was conducted in the absence of any commercial or financial relationships that could be construed as a potential conflict of interest.

Publisher's Note: All claims expressed in this article are solely those of the authors and do not necessarily represent those of their affiliated organizations, or those of the publisher, the editors and the reviewers. Any product that may be evaluated in this article, or claim that may be made by its manufacturer, is not guaranteed or endorsed by the publisher.

Copyright (๑ 2022 Kundu, Lin, Yoon, Parikh, Ruan, Kishan, Lee, Steinberg and Chang. This is an open-access article distributed under the terms of the Creative Commons Attribution License (CC BY). The use, distribution or reproduction in other forums is permitted, provided the original author(s) and the copyright owner(s) are credited and that the original publication in this journal is cited, in accordance with accepted academic practice. No use, distribution or reproduction is permitted which does not comply with these terms. 